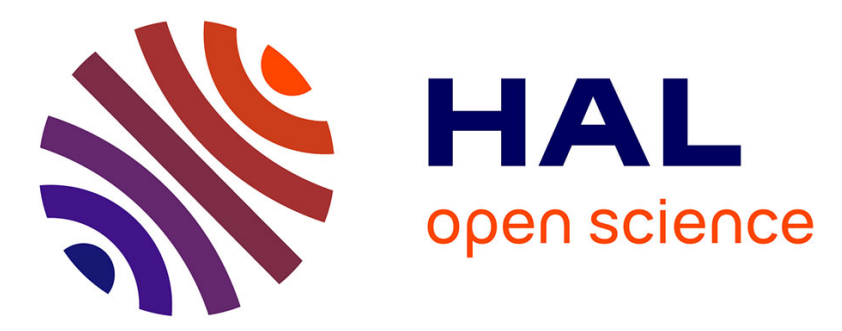

\title{
A Comparative Analysis of Attitude Estimation for Pedestrian Navigation with Smartphones
}

Thibaud Michel, Hassen Fourati, Pierre Genevès, Nabil Layaïda

\section{To cite this version:}

Thibaud Michel, Hassen Fourati, Pierre Genevès, Nabil Layaïda. A Comparative Analysis of Attitude Estimation for Pedestrian Navigation with Smartphones. IPIN 2015 - Indoor Positioning and Indoor Navigation, Oct 2015, Banff, Canada. pp.10, 10.1109/IPIN.2015.7346767 . hal-01194811

\section{HAL Id: hal-01194811 \\ https://hal.inria.fr/hal-01194811}

Submitted on 7 Sep 2015

HAL is a multi-disciplinary open access archive for the deposit and dissemination of scientific research documents, whether they are published or not. The documents may come from teaching and research institutions in France or abroad, or from public or private research centers.
L'archive ouverte pluridisciplinaire HAL, est destinée au dépôt et à la diffusion de documents scientifiques de niveau recherche, publiés ou non, émanant des établissements d'enseignement et de recherche français ou étrangers, des laboratoires publics ou privés. 


\title{
A Comparative Analysis of Attitude Estimation for Pedestrian Navigation with Smartphones
}

\author{
Thibaud Michel*†, Hassen Fourati ${ }^{\dagger}$, Pierre Genevès*, Nabil Layaïda* \\ ${ }^{*}$ Univ. Grenoble Alpes, LIG, CNRS, Inria, Grenoble, France \\ $\dagger$ Univ. Grenoble Alpes, GIPSA-Lab, F38000 Grenoble, France \\ CNRS, GIPSA-Lab, F-38000 Grenoble, France \\ Inria
}

\begin{abstract}
We investigate the precision of attitude estimation solutions in the context of Pedestrian Dead-Reckoning (PDR) with commodity smartphones and inertial/magnetic sensors. We propose a concise comparison and analysis of a number of attitude filtering methods in this setting. We conduct an experimental study with a precise ground truth obtained with a motion capture system. We precisely quantify the error in attitude estimation obtained with each filter which combines a 3-axis accelerometer, a 3-axis magnetometer and a 3-axis gyroscope measurements. We discuss the obtained results and analyse advantages and limitations of current technology for further PDR research.
\end{abstract}

Keywords-Attitude Estimation, Sensor Fusion, Inertial and Magnetic Sensors, Kalman Filter and Observer, Smartphone, Pedestrian Dead Reckoning.

\section{INTRODUCTION}

During the last years, the number of smartphone applications grew a lot and became more and more various. One of the main challenges to enable application diversity consists of determining the user location for indoor or outdoor contexts where GPS/GLONASS solutions are unavailable [1]. An embedded Pedestrian Navigation System (PNS) in a smartphone allows a large scale of applications: guide emergency first responders [2], museum tours, guide visually impaired, augmented reality [3], etc. Many systems have been developed to provide an indoor position using infrastructure supports like Wi-Fi and bluetooth but only few rely on embedded sensors [4]. Due to the technological evolution during the last years, our smartphones embed an Inertial Measurement Unit (IMU) composed of low-cost sensors: a 3-axis accelerometer, a 3-axis gyroscope, and a 3-axis magnetometer. This triad of sensors enables the use of Dead Reckoning algorithm, which consists in determining the current location by using a previously determined position. Such approach is usually used in aircrafts, submarines and cars. Pedestrian Dead Reckoning (PDR) is defined as follows: for each detected step, a new user position is built by computing the step-length and heading (Fig. 1a). Heading is the angle between the true North and user direction on the plane tangent to the Earth's surface (Fig. 1b). If the smartphone points to the motion direction, its attitude, expressed by yaw (or heading), pitch, and roll angles, is representing the orientation of pedestrian with respect to the Earth's frame.

This paper investigates the use of attitude estimation filters in the context of PDR with commodity smartphones. The impact of this estimation in PDR is shown in Fig. 2. The pair

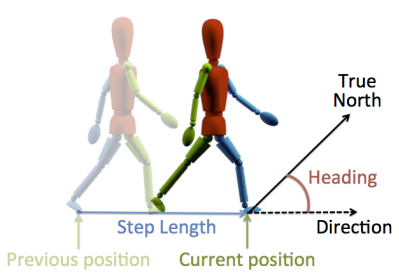

(a) Side View

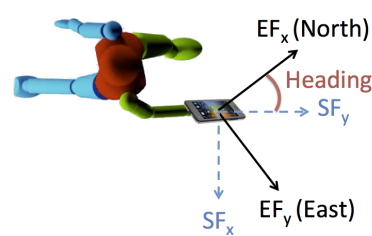

(b) Top View
Fig. 1. Pedestrian dead reckoning overview

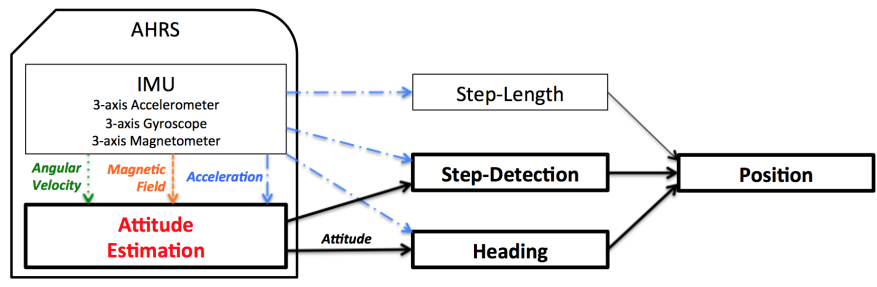

Fig. 2. Impact of attitude estimation on pedestrian dead reckoning

of IMU and attitude estimation block is called Attitude and Heading Reference System (AHRS).

\section{A. Related works}

The problem of attitude estimation is a well-known problem which has been extensively investigated, in particular for devices whose motion is rather well-characterized (with e.g. state equations). A survey of attitude estimation techniques for spacecrafts can be found in [5]. In comparison, the attitude estimation techniques for PDR based on smartphone's sensors still constitute a quite recent topic. One common aspect of all these methods is to combine measurements obtained from gyroscopes, accelerometers, and magnetometers. They usually consist of classical filtering methods using either Kalman filters (KFs) [6], extended Kalman filters (EKFs) [1], complementary filters [7], [8], [9], or observers [10].

One particular difficulty of PDR with smartphone's sensors is caused by various magnetic deviations in magnetometer data, that are commonly found in typical indoor environments [11]. These deviations are known to affect the precision of current estimation techniques. This problem has been investigated quite recently, through several approaches that support some forms of magnetic deviations [1], [10]. However, to the best of our knowledge, there is no comparative analysis of various 
estimation filters in the setting of PDR with smartphone's sensors. Authors typically evaluate their algorithms on their own ground truth, and still unrelated to others. As a result, it is very hard to grasp the advantages and limitations of each technique in our context.

\section{B. Contributions}

We propose a comparative analysis of recent attitude estimation filters using smartphone's sensors. We provide an experimental setup with a precise ground truth obtained through a motion capture system. We precisely quantify the attitude estimation error obtained with each technique. We pay attention at reproducibility of results 1 . We analyze and discuss the results we have obtained.

\section{Outline}

The rest of the paper is organized as follows. In Section II some useful notations, definitions and analysis for attitude estimation are given. In Section III, we present six main algorithms for attitude estimation and we discuss some key points of these algorithms. In Section IV we describe the experimental tests methodology, we report on the results of attitude estimation that we have obtained with each algorithm, and we discuss the results before concluding the paper in Section V.

\section{BACKGROUND FOR ATTITUDE ESTIMATION}

\section{A. Attitude representation}

The smartphone attitude in $\mathrm{PN}$ is determined when the axis orientation of the Smartphone Frame $S F\left(S F_{x}, S F_{y}, S F_{z}\right)$ is specified with respect to the Earth Frame $E F\left(E F_{x}, E F_{y}, E F_{z}\right)$ (see Fig. 3).

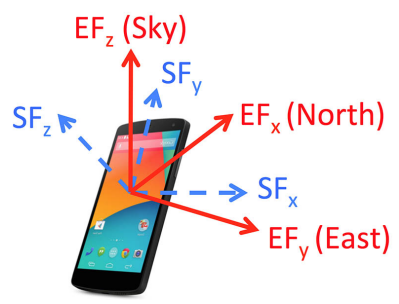

Fig. 3. The Smartphone-Frame SF (dashed line) and Earth-Frame EF (solid line).

The $S F_{x}$-axis is horizontal and points to the right, the $S F_{y}$ axis is vertical and points up and the $S F_{z}$-axis points towards the outside of the front face of the screen. The $E F_{y}$-axis points to the North. The $E F_{z}$-axis points to the sky perpendicular to the reference ellipsoid and the $E F_{x}$-axis completes the righthanded coordinate system, pointing East (ENU : East, North, Down). An other convention is often used by aerial Vehicles called NED for North, East and Down.

Based on the literature, the attitude can be expressed with three main different mathematical representations [12]. Euler angles (roll, pitch, yaw (or heading)), rotation matrix or quaternion.

${ }^{1}$ Benchmarks can be reproduced using datasets on our website: http://tyrex inria.fr/mobile/benchmarks-attitude
A unit-norm quaternion, which defines the rotation between $E F$ and $S F$, is defined as:

$$
q={ }_{E}^{S} q=\left[\begin{array}{l}
q_{0} \\
\vec{q}
\end{array}\right]=\left[\begin{array}{llll}
q_{0} & q_{1} & q_{2} & q_{3}
\end{array}\right]^{T} \in \mathbf{R}^{4},
$$

where $q_{0}$ and $\vec{q}$ being the scalar and the vector parts of the quaternion, respectively. We invite the reader to refer to [13] for more details about quaternion algebra.

Euler angles representation is composed of three main rotations: a rotation $\varphi$ around the $x$-axis (roll angle), a rotation $\theta$ around the $y$-axis (pitch angle) and a rotation $\psi$ around the $z$-axis (yaw angle). More details about Euler angles proprieties can be found in [14].

Rotation matrix for attitude estimation is a $3 \times 3$ matrix defining three unit vectors yielding a total of 9 parameters.

Each one of these representations has some disadvantages, Euler angles have the well known gimbal-lock problem [14], rotation matrix contains 9 elements to be determined and quaternion is less human understandable. Nevertheless, quaternion avoids the singularity problem involved in Euler angles and is featured with simple computation cost and is easy to be operated for a large number of applications, especially in the case of smartphone environment. In Section IV] the implemented algorithms under MATLAB are using the quaternion algebra [13]. A simple mathematical transformation between quaternion and Euler angles can be found in [14].

\section{B. Sensors measurements and calibration}

The sensors configuration of a smartphone is composed of a triad of MEMS (Micro-Electro-Mechanical Systems) sensors consisting of a 3-axis gyroscope, a 3-axis accelerometer and a 3-axis magnetometer. The outputs of these low-cost sensors are not perfect and suffer from several problems: noise, bias, scale factor, axis misalignment, axis non-orthogonality and local temperature. In the following, we will analyze the Allan variance [15] on each sensor embedded in the smartphone (Google Nexus 5) to provide an accurate models. The Allan variance analysis and results are similar to those proposed in other works [1], [16]. Let consider ${ }^{S} v$ as a $3 \times 1$ vector in the Smartphone frame and ${ }^{E} v$ as a $3 \times 1$ vector in the Earth frame.

1) Gyroscope: The 3-axis gyroscope measures the angular velocity of the smartphone in $\mathrm{rad} . \mathrm{s}^{-1}:{ }^{S} \omega=$ $\left[\begin{array}{lll}{ }^{S} \omega_{x} & S_{\omega_{y}} & { }^{S} \omega_{z}\end{array}\right]^{T}$. An Allan variance study [15] is applied to the gyroscope signal. The results are shown in Fig. 4 and three main noises are identified: an Angular Random Walk (ARW) given by the $-\frac{1}{2}$ slope part, a Bias Instability (BI) given by the 0 slope curve part and a Rate Random Walk (RRW) given by the $+\frac{1}{2}$ slope part. The widely used continuous time model for a gyroscope can be written such as:

$$
{ }^{S} \omega={ }^{S} \omega_{r}+{ }^{S} \omega_{b}+{ }^{S} \omega_{n}
$$

where

$S_{\omega} \quad$ is the angular rate measured by the gyroscope.

$S_{\omega_{r}} \quad$ is the true angular rate.

${ }^{S} \omega_{b}$ is the gyroscope bias, where its derivative ${ }^{S} \dot{\omega}_{b}$ is modeled by a random walk noise ${ }^{S} \dot{\omega}_{b}={ }^{S} \omega_{b_{n}}$, its 
standard deviation (BI) is noted ${ }^{S} \sigma_{\omega_{b n}}$. The gyroscope bias leads after integration (see Eq. (7)) to an angular drift, increasing linearly over time.

${ }^{S} \omega_{n}$ is the gyroscope white noise, its standard deviation (ARW) is noted ${ }^{S} \sigma_{\omega_{n}}$.

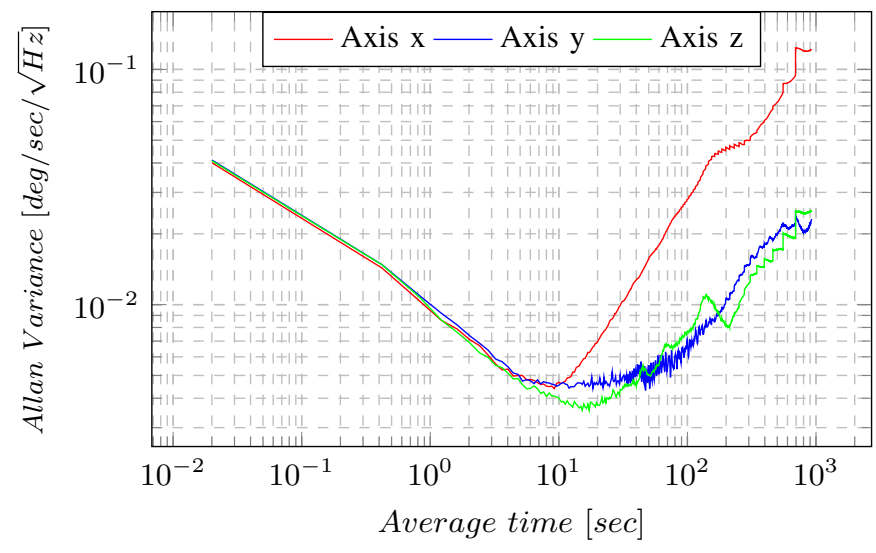

Fig. 4. Allan variance of gyroscope signal.

Since the use of only gyroscope is not enough for attitude estimation, other complementary sensors are used such as accelerometers and magnetometers to compensate this drift. The accelerometer allows the correction of the pitch and roll angles whereas the magnetometer improves the yaw angle. So, how to pertinently combine inertial and magnetic sensor measurements, is the key question to be solved when we are facing to an attitude estimation problem.

2) Accelerometer: The 3-axis accelerometer measures the sum of the gravity and external acceleration of the smartphone in $m . s^{-2}:{ }^{S} a=\left[\begin{array}{lll}{ }^{S} a_{x} & { }^{S} a_{y} & { }^{S} a_{z}\end{array}\right]^{T}$. In the same way as the gyroscope, we used the Allan variance on the accelerometer signal. The results are shown in Fig. 5 and three main noises are identified: a Velocity Random Walk (VRW) given by the $-\frac{1}{2}$ slope part, a Bias Instability (BI) given by the 0 slope curve part and a Correlated Noise $(\mathrm{CN})$ given by the oscillations (mostly on $\mathrm{x}$-axis and z-axis). The continuous time model for accelerometer can be written such as:

$$
{ }^{s} a={ }^{S} a_{r}+{ }^{S} a_{b}+{ }^{S} a_{n},
$$

where

${ }^{S} a$ is the sum of the gravity and external acceleration of the body measured by the accelerometer.

${ }^{S} a_{r}$ is the true sum of the gravity and external acceleration of the body.

${ }^{S} a_{b}$ is the accelerometer bias, where its derivative ${ }^{S} \dot{a}_{b}$ is modeled by a Gauss-Markov noise: ${ }^{S} \dot{a}_{b}=\beta^{S} a_{b}+{ }^{S} a_{b_{n}}$, the standard deviation of ${ }^{S} a_{b_{n}}$ (BI) is noted ${ }^{S} \sigma_{a_{b_{n}}}$.

${ }^{S} a_{n}$ is the accelerometer white noise, its standard deviation (VRW) is noted ${ }^{S} \sigma_{a_{n}}$.

A uncalibrated accelerometer in a static phase provides a magnitude of acceleration close to $g$. In [17], the authors provide an accelerometer calibration algorithm based on a minimum of 7 static phases. This calibration allows to remove the bias and misalignment by normalizing the acceleration vector in multiple smartphone orientations. Finally the acceleration magnitude is close to $g$.

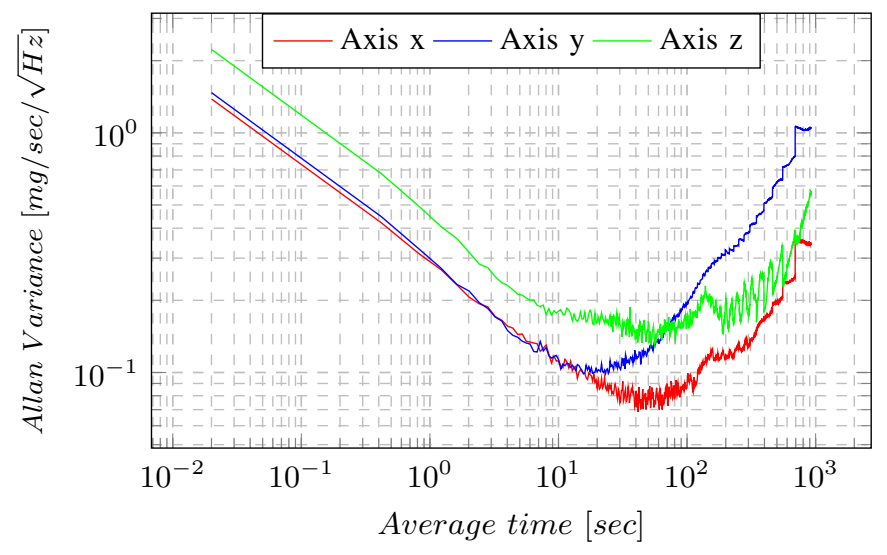

Fig. 5. Allan variance of accelerometer signal.

3) Magnetometer: The 3-axis magnetometer measures the magnetic field of the smartphone in micro-tesla $(\mu T):{ }^{S} m=$ $\left.{ }^{S} m_{x}{ }^{S} m_{y}{ }^{S} m_{z}\right]^{T}$. The Earth's magnetic field can be modeled by a dipole and follows basic laws of magnetic fields. At any location, the Earth's magnetic field can be represented by a three-dimensional vector and its intensity varies from $25 \mu T$ to $65 \mu T$. The National Geospatial-Intelligence Agency (NGA) and the United Kingdoms Defence Geographic Centre (DGC) provide a World Magnetic Model (WMM) [18] every 5-years as shown in Fig. 6 Declination is used to know the angle between the Magnetic North and Geographic North, while inclination and intensity are used to build the reference vector.

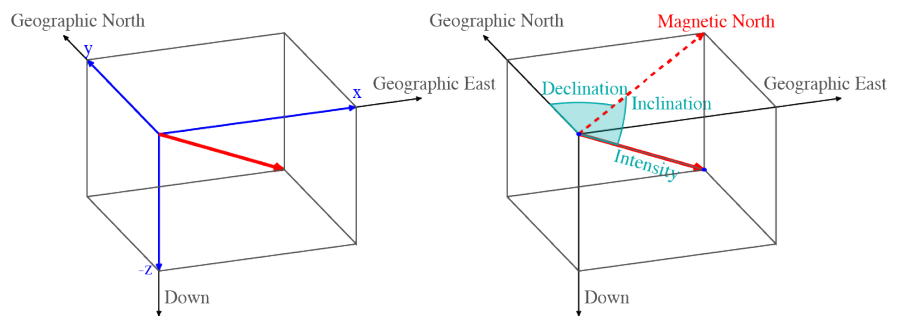

Fig. 6. Earth's magnetic field representation

The Allan variance is used on magnetometer signal and the results are shown in Fig. 7 where three main noises are identified: an Angle Random Walk (ARW) given by the $-\frac{1}{2}$ slope part, a Bias Instability (BI) given by the 0 slope curve part and a Correlated Noise $(\mathrm{CN})$ given by the oscillations. The continuous time model for magnetometer can be written such as:

$$
{ }^{S} m={ }^{S} m_{r}+{ }^{S} m_{b}+{ }^{S} m_{n},
$$

where

${ }^{S} m$ is the magnetic field measured by the magnetometer.

${ }^{S} m_{r}$ is the true magnetic field.

${ }^{S} m_{b}$ is the magnetometer bias, where its derivative ${ }^{S} \dot{m}_{b}$ is modeled by a Gauss-Markov noise: ${ }^{S} \dot{m}_{b}=\beta^{S} m_{b}+$ $S_{m_{b_{n}}}$, the standard deviation of ${ }^{S} m_{b_{n}}$ (BI) is noted ${ }^{S} \sigma_{m_{b_{n}}}$. 
${ }^{S} m_{n}$ is the magnetometer white noise, its standard deviation (ARW) is noted ${ }^{S} \sigma_{m_{n}}$.

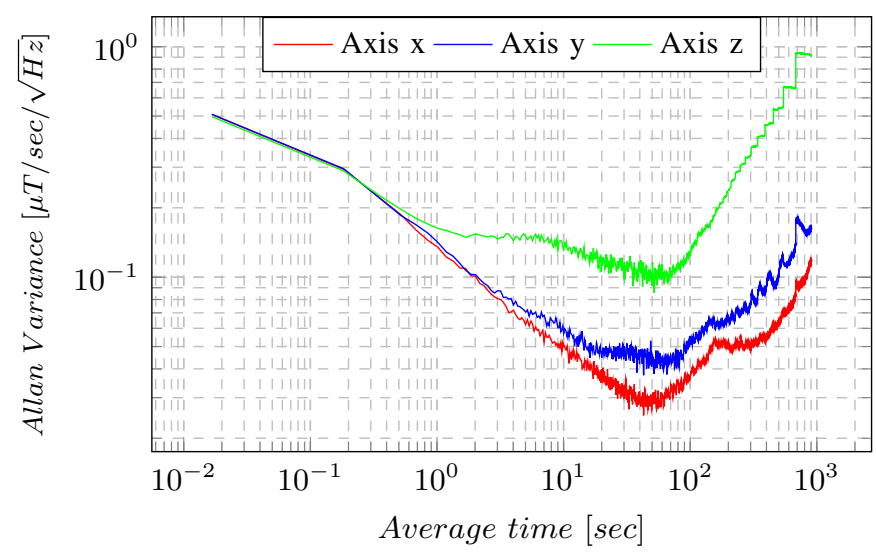

Fig. 7. Allan variance of magnetometer signal.

Unfortunately, the magnetometer do not measure only the Earth's magnetic field. Most of the time in indoor environments, we are in presence of magnetic dipoles which perturb the measure of Earth's magnetic field. These perturbations can be caused by electromagnetic devices (speakers, magnets), manmade structures (walls, floors) or other ferromagnetic objects like belts, keys... For example, a speaker of a smartphone has a field of about $2000 \mu T$ (30 times more than the Earth's magnetic field). A study in [11] categorizes the environmental characteristics with respect to the magnetic deviations. Magnetic perturbations are categorized in two groups: hard and soft iron distortions. Hard iron distortions are caused by ferromagnetic materials in the smartphone frame SF (e.g. speaker for a smartphone). Soft iron distortions are caused by objects that produce a magnetic field (buildings walls, machines...) in the Earth frame EF. In a context free from magnetic interferences, hard and soft iron distortions can be corrected at the same time by normalizing the magnetic field vector in a multiple smartphone orientations [19], [20].

\section{ATTITUDE ESTIMATION ALGORITHMS OVERVIEW}

For more clarity to the reader, we present in this section the overall design of attitude estimation filters we need to compare. The selected filters [1], [6], [7], [8], [9], [10] from the literature have been designed for different applications and based on Kalman filter, complementary filter and observer theories.

\section{A. Filters design}

The six algorithms are presented with a common notations on quaternion and sensors readings as described in Section II and also below:

$\hat{v}=\left[\begin{array}{lll}\hat{v}_{x} & \hat{v}_{y} & \hat{v}_{z}\end{array}\right]^{T}$ is used for the estimated vector $v$,

$k_{A c c}, k_{G y r}, k_{M a g}$ are the weight that are used to most trust a sensor that another,

$q_{e}, \omega_{e} \quad$ are the quaternion and angular rate errors, $\Delta t \quad$ is the time difference between 2 epochs.

In order to estimate $q$, all presented algorithms use two reference vectors ${ }^{E} a$ and ${ }^{E} m$.
In a perfect case (free of noise), the relation between ${ }^{E} a$ and $S_{a}$ is described as following:

$$
{ }^{S} a_{q}=q^{-1} \otimes{ }^{E} a_{q} \otimes q,
$$

where

$\otimes \quad$ is the quaternion multiplication [13].

${ }^{S} a_{q}$ is the quaternion form of ${ }^{S} a$, which can be written such as: ${ }^{S} a_{q}=\left[\begin{array}{llll}0 & { }^{S} a_{x} & { }^{S} a_{y} & { }^{S} a_{z}\end{array}\right]^{T}$.

${ }^{E} a_{q}$ is the quaternion form of ${ }^{E} a$ (such as ${ }^{S} a_{q}$ ). In static cases, ${ }^{E} a=\left[\begin{array}{lll}0 & 0 & g\end{array}\right]^{T}$ where $g$ is the acceleration due to the gravity at the Earth's surface $\left(g \approx 9.8 \mathrm{~m} . \mathrm{s}^{-2}\right)$.

In a perfect case (free of noise and magnetic deviation), the relation between ${ }^{E} m$ and ${ }^{S} m$ is described as following:

$$
{ }^{S} m_{q}=q^{-1} \otimes{ }^{E} m_{q} \otimes q,
$$

where

${ }^{S} m_{q}$ is the quaternion form of ${ }^{S} m$, which can be written such as: ${ }^{S} m_{q}=\left[\begin{array}{llll}0 & { }^{S} m_{x} & { }^{S} m_{y} & { }^{S} m_{z}\end{array}\right]^{T}$.

${ }^{E} m_{q}$ is the quaternion form of ${ }^{E} m$. In the absence of magnetic deviations, ${ }^{E} m$ can be calculated by using the WMM [18].

The well-known kinematic equation of rigid body which is defined from angular velocity measurements from a gyroscope to describe the variation of the attitude in term of quaternion can be written such as:

$$
\dot{q}=\frac{1}{2} q \otimes{ }^{S} \omega_{q},
$$

where ${ }^{S} \omega_{q}$ is the quaternion form of ${ }^{S} \omega$.

Algorithm 1 - Mahony et al. [7]

Explicit Complementary Filter

$$
\begin{aligned}
& { }^{S} \hat{a}_{q, t}=\hat{q}_{t-1}^{-1} \otimes{ }^{E} a_{q, t} \otimes \hat{q}_{t-1} \\
& { }^{S} \hat{m}_{q, t}=\hat{q}_{t-1}^{-1} \otimes{ }^{E} m_{q, t} \otimes \hat{q}_{t-1} \\
& { }^{S} \omega_{m e s, t}=\left[\begin{array}{ll}
\left.{ }^{S} a_{t} \times{ }^{S} \hat{a}_{t}\right]+\left[{ }^{S} m_{t} \times{ }^{S} \hat{m}_{t}\right.
\end{array}\right] \\
& { }^{S} \dot{\hat{\omega}}_{b, t}=-k_{i}{ }^{S} \omega_{m e s, t} \\
& { }^{S} \hat{\omega}_{r, q, t}={ }^{S} \omega_{q, t}-\left[\begin{array}{ll}
0 & { }^{S} \hat{\omega}_{b, t}
\end{array}\right]+\left[\begin{array}{ll}
0 & k_{p}{ }^{S} \omega_{m e s, t}
\end{array}\right]
\end{aligned}
$$

$k_{p}$ and $k_{i}$ are proportional and integral adjustable gains (see Eq. 7]).

$$
\dot{\hat{q}}_{t}=\frac{1}{2} \hat{q}_{t-1} \otimes{ }^{S} \hat{\omega}_{r, q, t}
$$

Principles : This filter is an Explicit Complementary Filter designed for aerial vehicles. The algorithm calculates the error by cross multiplying the measured and estimated vectors (acceleration and magnetic field), then this error allows to correct the gyroscope bias. 
Algorithm 2 - Madgwick et al. [8]

Gradient Descent based Orientation Filter

${ }^{E} \hat{h}_{q, t}=\hat{q}_{t-1} \otimes{ }^{S} m_{q, t} \otimes \hat{q}_{t-1}^{-1}$

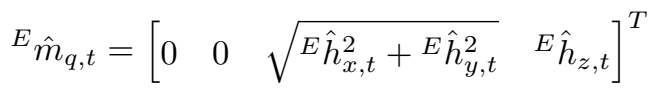

$F_{t}=\left[\begin{array}{c}\hat{q}_{t-1}^{-1} \otimes{ }^{E} a_{q, t} \otimes \hat{q}_{t-1}-{ }^{S} a_{q, t} \\ \hat{q}_{t-1}^{-1} \otimes{ }^{E} \hat{m}_{q, t} \otimes \hat{q}_{t-1}-{ }^{S} m_{q, t}\end{array}\right]$

$\hat{q}_{e, t}=J_{t}^{T} F_{t}$, where $J_{t}$ is the Jacobian matrix of $F_{t}$ (see [8])

${ }^{S} \hat{\omega}_{e, t}=2 \hat{q}_{t-1} \otimes \hat{q}_{e, t}$

$S \dot{\hat{\omega}}_{b, t}={ }^{S} \omega_{e, t}$

${ }^{S} \hat{\omega}_{t}={ }^{S} \omega_{t}-\zeta^{S} \omega_{b, t}$, where $\zeta$ is the integral gain.

$\dot{\hat{q}}_{t}=\frac{1}{2} \hat{q}_{t-1} \otimes S \hat{\omega}_{q, t}-\beta \frac{\hat{q}_{e, t}}{\left\|\hat{q}_{e, t}\right\|}$

$\beta$ is the divergence rate of $q_{t}$ expressed as the magnitude of a quaternion derivative corresponding to the gyroscope measurement error.

Principles : This filter is a Gradient Descent (GD) based algorithm designed for pedestrian navigation. The quaternion error from the GD algorithm provides also a gyro drift compensation.

Algorithm 3 - Fourati [9]

Complementary Filter Algorithm

$$
\begin{aligned}
& { }^{S} \hat{a}_{q, t}=\hat{q}_{t-1}^{-1} \otimes{ }^{E} a_{q, t} \otimes \hat{q}_{t-1} \\
& { }^{S} \hat{m}_{q, t}=\hat{q}_{t-1}^{-1} \otimes{ }^{E} m_{q, t} \otimes \hat{q}_{t-1} \\
& X=-2\left[\begin{array}{ll}
{\left[{ }^{S} \hat{a}_{t} \times\right.} & {\left[{ }^{S} \hat{m}_{t} \times\right.}
\end{array}\right]^{T} \text {, where: } \\
& {\left[v^{\times}\right]=\left[\begin{array}{ccc}
0 & -v_{z} & v_{y} \\
v_{z} & 0 & -v_{x} \\
-v_{y} & v_{x} & 0
\end{array}\right]} \\
& K=\left[X^{T} X+\lambda I_{3 \times 3}\right]^{-1} X^{T} \text {, with } \lambda=10^{-5} \\
& \hat{q}_{e, t}=\left[\begin{array}{c}
1 \\
K\left[\begin{array}{c}
{ }^{S} a_{t}-{ }^{S} \hat{a}_{t} \\
{ }^{S} m_{t}-{ }^{S} \hat{m}_{t}
\end{array}\right]
\end{array}\right] \\
& \dot{\hat{q}}_{t}=\frac{1}{2} \hat{q}_{t-1} \otimes{ }^{S} \hat{\omega}_{q, t} \otimes \beta \hat{q}_{e, t}
\end{aligned}
$$

Principles : This filter is a mix between a GD algorithm and the quadratic approach of Marquardt designed for foot-mounted devices. In this algorithm, there is no gyroscope drift compensation and only one adjustable gain is used. From author recommendations, we chose to put $\beta=5$ when $\left\|{ }^{S} a\right\|-g<0.03$ and $\beta=0.5$ for others cases. ${ }^{E} m$ used by Fourati is defined as follows: ${ }^{E} m=\left[\begin{array}{lll}0 & \cos (i n c) & -\sin (\text { inc })\end{array}\right]^{T}$ expressed in ENU coordinates where inc is the inclination at the smartphone location defined by WMM [18].

$$
\begin{aligned}
& \text { Algorithm } 4 \text { - Martin et al. [10] } \\
& \text { Invariant Nonlinear Observers } \\
& { }^{S_{c}}=\left[{ }^{S} a \times{ }^{S} m\right] \quad{ }^{E} c=\left[{ }^{E} a \times{ }^{E} m\right] \\
& { }^{S} d=\left[{ }^{S} c \times{ }^{S} a\right] \quad{ }^{E} d=\left[{ }^{E} c \times{ }^{E} a\right] \\
& E_{t}=\left[\begin{array}{c}
E_{A_{t}} \\
E_{C_{t}} \\
E_{D_{t}}
\end{array}\right]=\left[\begin{array}{c}
{ }^{E} a_{q}-\frac{1}{\hat{o}_{s}} \hat{q}_{t-1} \otimes{ }^{S} a_{q, t} \otimes \hat{q}_{t-1}^{-1} \\
{ }^{E} c_{q}-\frac{1}{\hat{c}_{s}} \hat{q}_{t-1} \otimes{ }^{S} c_{q, t} \otimes \hat{q}_{t-1}^{-1} \\
{ }^{E} d_{q}-\frac{1}{\hat{a}_{s} \times \hat{c}_{s}} \hat{q}_{t-1} \otimes{ }^{S} d_{q, t} \otimes \hat{q}_{t-1}^{-1}
\end{array}\right] \\
& L E_{t}={\frac{k_{a}}{g^{2}}}^{2} a \times E_{A_{t}}+\frac{k_{c}}{\left.{ }^{E} m_{y} g\right)^{2}}{ }^{E} c \times E_{C_{t}}+\frac{k_{d}}{\left({ }^{E} m_{y} g^{2}\right)^{2}}{ }^{E} d \times E_{D_{t}} \\
& M E_{t}=-k_{\sigma} L E_{t} \\
& N E_{t}=\frac{k_{n}}{k_{a}+k_{d}}\left(\frac{k_{A}\left\langle E_{A_{t}}, E_{A_{t}}-{ }^{E} a\right\rangle}{g^{2}}+\frac{k_{D}\left\langle E_{D_{t}}, E_{D_{t}}-{ }^{E} d\right\rangle}{\left({ }^{E} m_{y} g^{2}\right)^{2}}\right) \\
& O E_{t}=\frac{k_{o}}{k_{a}+k_{d}}\left(\frac{k_{C}\left\langle E_{C_{t}}, E_{C_{t}}-{ }^{E} c\right\rangle}{\left.{ }^{E} m_{y} g\right)^{2}}+\frac{k_{D}\left\langle E_{D_{t}}, E_{D_{t}}-{ }^{E} d\right\rangle}{\left({ }^{E} m_{y} g^{2}\right)^{2}}\right) \\
& \text { with } k_{a}, k_{c}, k_{d}, k_{\sigma}, k_{n}, k_{o}>0 \\
& \dot{\hat{q}}_{t}=\frac{1}{2} \hat{q}_{t-1} \otimes\left({ }^{S} \omega_{q, t}-{ }^{S} \hat{\omega}_{b, q, t}\right)+L E_{t} \otimes \hat{q}_{t-1} \\
& \dot{\hat{\omega}}_{b}=\hat{q}_{t-1}^{-1} \otimes M E_{t} \otimes \hat{q}_{t-1} \\
& \dot{\hat{a}}_{s}=\hat{a}_{s} \times N E_{t} \\
& \dot{\hat{c}}_{s}=\hat{c}_{s} \times O E_{t}
\end{aligned}
$$

Principles : This filter is an observer with a new geometrical structure from an engineering viewpoint. Authors introduce new measures: ${ }^{S} c$ is based on the product of ${ }^{S} a$ and ${ }^{S} m,{ }^{S} d$ is the product of ${ }^{S} c$ and ${ }^{S} a$. This approach does not require to know all components of ${ }^{E} m .{ }^{E} m_{y}$ is the second component of $E_{m}$. An accelerometer, gyroscope and magnetometer biases are estimated at every sample.

\section{Algorithm 5 - Choukroun et al. [6]}

\section{Linear Kalman Filter}

Time update:

$$
\begin{aligned}
& \Omega_{t}=\left[\begin{array}{cc}
0 & -{ }^{S} \omega_{t}^{T} \\
{ }^{S} \omega_{t} & -\left[{ }^{S} \omega_{t} \times\right]
\end{array}\right] \Delta t \\
& \hat{q}_{t}^{-}=\Phi_{t} \times q_{t-1}^{T} \text {, with } \Phi_{t}=\exp \left(\frac{1}{2} \Omega_{t}\right) \\
& \Xi_{t}=\left[\begin{array}{c}
-\hat{\overrightarrow{q_{t}}} \\
-\left[\hat{\overrightarrow{q_{t}}} \times\right]+{\hat{q_{0}}}_{0_{t}} \times I_{3 \times 3}
\end{array}\right] \\
& Q_{f, t}=\frac{1}{4} \Xi_{t} Q_{g} \Xi_{t}^{T} \Delta t
\end{aligned}
$$


$P_{t}^{-}=\Omega_{t} P_{t-1} \Omega_{t}^{T}+Q_{f, t}$

$Q_{g}$ is the covariance matrix of state.

Measurement update:

$$
\begin{aligned}
& s_{a_{t}}=\frac{1}{2}\left({ }^{S} a_{t}+{ }^{E} a\right) \quad d_{a_{t}}=\frac{1}{2}\left({ }^{S} a_{t}-{ }^{E} a\right) \\
& s_{m_{t}}=\frac{1}{2}\left({ }^{S} m_{t}+{ }^{E} m\right) \quad d_{m_{t}}=\frac{1}{2}\left({ }^{S} m_{t}-{ }^{E} m\right) \\
& H_{a_{t}}=\left[\begin{array}{cc}
0 & -d_{a_{t}}^{T} \\
d_{a_{t}} & -\left[s_{a_{t}}^{\times}\right.
\end{array}\right] \quad H_{m_{t}}=\left[\begin{array}{cc}
0 & -d_{m_{t}}^{T} \\
d_{m_{t}} & -\left[s_{m_{t}}^{\times}\right]
\end{array}\right] \\
& \left.\bar{H}_{t}=\left[\begin{array}{l}
H_{m_{t}} \\
H_{a_{t}}
\end{array}\right] \quad \begin{array}{c}
0_{4 \times 4}\left(E_{1_{t}}{ }^{S} \sigma_{a_{n}} E_{1_{t}}^{T}\right)+\lambda I_{3 \times 3}
\end{array}\right] \\
& E_{1_{t}}=\left[\begin{array}{c}
-\hat{\vec{q}}_{t}^{-} \\
-\left[\hat{\vec{q}}_{t}^{-} \times{ }^{\times}\right]+\hat{q}_{0_{t}}^{-} I_{3 \times 3}
\end{array}\right] \\
& \bar{R}_{t}=\left[\begin{array}{c}
\frac{1}{4}\left(E_{1_{t}} \sigma_{m a g} E_{1_{t}}^{T}\right)+\lambda I_{3} \\
0_{4 \times 4}
\end{array}\right. \\
& K_{t}=P_{t}^{-} \bar{H}_{t}^{T}\left(\bar{H}_{t} P_{t}^{-} \bar{H}_{t}^{T}+\bar{R}_{t}\right)^{-1} \\
& \hat{q}_{t}=\hat{q}_{t}^{-}-K_{t} \bar{H}_{t} \hat{q}_{t}^{-} \\
& P_{t}=\left(I_{4 \times 4}-K_{t} \bar{H}_{t}\right) P_{t}^{-}\left(I_{4 \times 4}-K_{t} \bar{H}_{t}\right)+K_{t} \bar{R}_{t} K_{t}^{T}
\end{aligned}
$$

Principles : This filter is the first one that provides a linearization of measurement equations. Then a Kalman Filter is applied and guarantees a global convergence.

Algorithm 6 - Renaudin et al. [1]

EKF with opportunistic updates

State Vector:

$$
x=\left[\begin{array}{lll}
q_{t} & q_{\omega_{b}, t} & a_{b_{t}}
\end{array}\right]^{T}
$$

Time update:

$$
\begin{aligned}
q_{\omega, t}= & {\left[\begin{array}{c}
\cos \left(\frac{1}{2}\left\|S \omega_{t}\right\| \Delta t\right) \\
\sin \left(\frac{1}{2}\left\|{ }^{S} \omega_{t}\right\| \Delta t\right) \frac{S_{\omega_{t}}}{\left\|S \omega_{t}\right\|}
\end{array}\right] } \\
\hat{q}_{\omega, t}= & q_{\omega, t}-\hat{q}_{\omega_{b}, t} \\
\hat{q}_{t}=\hat{q}_{t-1} \otimes \hat{q}_{\omega, t} & \\
x_{t}=\hat{x}_{t}+\delta x_{t} & {\left[\begin{array}{ccc}
C\left(\hat{q}_{\omega, t}\right) & -M\left(\hat{q}_{t}\right) & 0 \\
0 & I_{3 \times 3} & 0 \\
0 & 0 & I_{3 \times 3}-\sigma_{a_{b_{n}}} \Delta t
\end{array}\right] \delta x_{t-1} } \\
\delta x_{t}= & {\left[\begin{array}{ccc}
-M\left(\hat{q}_{t}\right) & 0 & 0 \\
0 & I_{3 \times 3} \Delta t & 0 \\
0 & 0 & I_{3 \times 3} \Delta t
\end{array}\right]\left[\begin{array}{c}
q_{\omega_{n}} \\
q_{\omega_{b_{n}}} \\
a_{n}
\end{array}\right] }
\end{aligned}
$$

$q_{\omega_{n}}$ and $q_{\omega_{b_{n}}}$ are the $q_{\omega}$ noise and noise of bias.
Measurement update (only during QSF):

$$
\begin{aligned}
& \delta z_{m, q, t}={ }^{E} m_{q, t}-\hat{q}_{t} \otimes{ }^{S} m_{q, t} \otimes \hat{q}_{t}^{-1} \\
& \delta z_{m, t}=\left[h_{1}\left(\hat{q}_{t},{ }^{E} m\right) \quad 0 \quad 0\right] \delta x-h_{2}\left(\hat{q}_{t}\right) m_{n} \\
& { }^{S} \hat{m}_{q, t}=\hat{q}_{\omega, t-1} \otimes{ }^{S} m_{q, t-1} \otimes \hat{q}_{\omega, t-1}^{-1} \\
& \delta z_{M A R U, t}={ }^{S} m_{t}-{ }^{S} \hat{m}_{t} \\
& \delta z_{M A R U, t}=\left[\begin{array}{lll}
0 & -h_{3}\left(\hat{q}_{\omega, t},{ }^{S} \hat{m}_{t}\right) & 0
\end{array}\right] \delta x \\
& +\left[\begin{array}{ll}
I_{3 \times 3} & -h_{2}\left(\hat{q}_{\omega, t}^{-1}\right)
\end{array}\right]\left[\begin{array}{c}
m_{n_{t}} \\
m_{n_{t-1}}
\end{array}\right] \\
& \delta z_{a, q, t}={ }^{E} a_{q}-\hat{q}_{t} \otimes\left({ }^{S} a_{q, t}-\hat{a}_{b, t}\right) \otimes \hat{q}_{t}^{-1} \\
& \delta z_{a, t}=\left[h_{1}\left(\hat{q}_{t},{ }^{E} a\right) \quad 0 \quad-h_{2}\left(\hat{q}_{t}\right)\right] \delta x_{t}-h_{2}\left(\hat{q}_{t}\right) a_{n} \\
& S_{\hat{a}_{q, t}}=\hat{q}_{\omega, t-1}^{-1} \otimes\left({ }^{S} a_{q, t-1}-\hat{a}_{b, t-1}\right) \otimes \hat{q}_{\omega, t-1} \\
& \delta z_{A G U, t}={ }^{S} a_{t}-\left({ }^{S} \hat{a}_{t}+\left(I_{3 \times 3}-\sigma_{a_{b_{n}}} \Delta t\right) \hat{a}_{b_{t-1}}\right)
\end{aligned}
$$

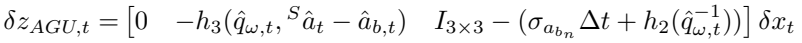

$$
\begin{aligned}
& +\left[\begin{array}{ll}
I_{3 \times 3} & -h_{2}\left(\hat{q}_{\omega, t}^{-1}\right)
\end{array}\right]\left[\begin{array}{c}
a_{n_{t}} \\
a_{n_{t-1}}
\end{array}\right]
\end{aligned}
$$

$h_{1}, h_{2}, h_{3}, C, M$ are defined in Renaudin's paper [1].

Principles : This filter is an Extended Kalman Filter designed for PDR algorithm. It does not use directly the gyroscope model (see Eq. (2)). The gyroscope signal is interpreted as a rotation between two successive epochs called $q_{\omega}$. An accelerometer gradient update (AGU) is applied only during Quasi-Static Field (QSF) periods. The same technique is used for magnetic angular rate update (MARU) during QSF. Apart from QSF periods, an integration on gyroscope measurements is processed (see Eq. (7). The detector of QSF is not described here.

\section{B. Discussions}

All algorithms compared here share the same objective (finding an estimation of $q$ ) but rely on slightly different methods for this purpose: gradient descent, cross product error, Kalman filters, etc. From theory, there is no reason why one method would always perform better than the others, hence the interest of our experimental analysis. We can however comment on a few assumptions used by several methods, which might be questionable when used in our context.

External acceleration. Algorithms used by Mahony, Madgwick, Martin and Choukroun rely on a common assumption: external acceleration of the smartphone is negligible. However, when used in this particular context of smartphone carried by a pedestrian, this assumption is questionable. Specifically, the relation between ${ }^{S} a$ and ${ }^{E} a$ given by Eq. (5) is true only if no external acceleration is applied on the smartphone. Mahony, Madgwick, Martin and Choukroun consider external acceleration as negligible, but in some cases for a pedestrian, magnitude of smartphone external acceleration can rise up to $5 m . s^{-2}$. Fourati takes it into account by adjusting gain according 
to small or large accelerations. Renaudin's approach is close to Fourati's one, a filter is applied only when $m a(\|S a\|-g)<\lambda$ where $m a$ is a moving-average, otherwise an integration on gyroscope is computed (see Eq. (7)).

Magnetic deviations. Algorithms consider different techniques for dealing with magnetic deviations. Specifically, different approaches are used for modelling ${ }^{E} m$. Choukroun and Fourati used a vector built from inclination described in Section II-B3, in other words they do not consider magnetic deviations. Another approach considers small changes in magnetic field vector in the $\mathrm{EF}$, it allows to use magnetic field vector of last sample as reference. Madgwick, Mahony and Martin used this approach. Renaudin uses this last approach and combines it with gyroscope integration (see Eq. (7)) when there are changes in magnetic field vector. Among all algorithms compared here, only Martin's one prevents magnetic deviations from modifying pitch and roll values, only yaw is impacted. We will verify experimentally this claim in the next section.

Measurement models. Sensors are not considered by algorithms exactly as described in Section II-B Table I shows which noise and which bias are considered by algorithms.

TABLE I. SENSORS' BIASES AND NOISES CONSIDERATION IN EACH ALGORITHM

\begin{tabular}{|r|c|c|c|c|c|c|}
\hline & \multicolumn{2}{|c|}{ Gyroscope } & \multicolumn{2}{c|}{ Accelerometer } & \multicolumn{2}{c|}{ Magnetometer } \\
& Bias & Noise & Bias & Noise & Bias & Noise \\
\hline Mahony & $\mathrm{X}$ & & & & & \\
\hline Madgwick & $\mathrm{X}$ & & & & & \\
\hline Fourati & & & & & & \\
\hline Martin & $\mathrm{X}$ & & $\mathrm{X}$ & & $\mathrm{X}$ & \\
\hline Choukroun & $\mathrm{X}^{2}$ & $\mathrm{X}$ & & $\mathrm{X}$ & & $\mathrm{X}$ \\
\hline Renaudin & $\mathrm{X}$ & $\mathrm{X}$ & $\mathrm{X}$ & $\mathrm{X}$ & & $\mathrm{X}$ \\
\hline
\end{tabular}

The number of filters parameters can become a problem because they are hard to estimate and depend on sensors quality or sensors dynamics. Table $\mathrm{II}$ shows the number of adjustable parameters in each algorithm excluding noise and bias. Values of parameters used for Section $[\mathrm{IV}$ are mostly extracted from authors' papers:

Mahony: $k_{p}=1$ and $k_{i}=0.3$.

Madgwick: $\beta=0.08, \zeta=0.003$ when time $>10 \mathrm{~s}$ and $\zeta=0$ during the first ten seconds.

Fourati: It has no adjustable parameters since the gain is dynamic.

Martin: $k_{a}=0.7, k_{c}=0.1, k_{d}=0.01, k_{n}=0.01, \quad k_{o}=$ $0.01, k_{\sigma}=0.05$.

Choukroun: The only parameters are noises of sensors.

Renaudin: Same parameters than Choukroun for noises but for acceleration and magnetic field QSF detectors, thresholds are respectively $\lambda_{A c c}=3.92 m . s^{-2}$ and $\lambda_{M a g}=6 \mu T$.

TABLE II. NUMBER OF PARAMETERS OF EACH ALGORITHM

\begin{tabular}{|c|c|c|c|c|c|}
\hline Mahony & Madgwick & Fourati & Martin & Choukroun & Renaudin \\
\hline 2 & 2 & 0 & 6 & 0 & 2 \\
\hline
\end{tabular}

Globally, Kalman filters are easier to parametrize because noises values are only extracted from Allan variance.

\footnotetext{
${ }^{2}$ This bias has not been implemented in our version.
}

\section{COMPARATIVE ANALYSIS}

\section{A. Experimental context}

Multiple experiences have been conducted with several smartphones (including LG Nexus 5, iPhone 5, Sony Xperia Z) and we obtained very similar results. A further study has been made with LG Nexus 5, thanks to a homemade log application 3 . Internal sensors are really cheap and cost at most $3 €$. The first one is a 6-axis InvenSense MPU6515 accelerometer and gyroscope. The second one is a 3-axis AKM AK8963 magnetometer. InvenSense sensor can monitor activity up to $200 \mathrm{~Hz}$ while AKM one only to $60 \mathrm{~Hz}$. For the purpose of aligning timestamps of magnetic field data with data obtained from accelerometer and gyroscope, we used a linear extrapolation, in order to keep the focus on a real-time algorithm, interpolation is unallowable here. We choose to align data at $200 \mathrm{~Hz}$ but similar results can be obtained until a sampling at $50 \mathrm{~Hz}$.

Tests have been made in a $10 \mathrm{~m} \times 10 \mathrm{~m}$ square motion la ${ }^{4}$ In this room, we observed that the magnetic field is almost homogeneous from a subplace to another (variations are less than $3 \mu T$ : see Fig. 8), and with negligible variations over time.

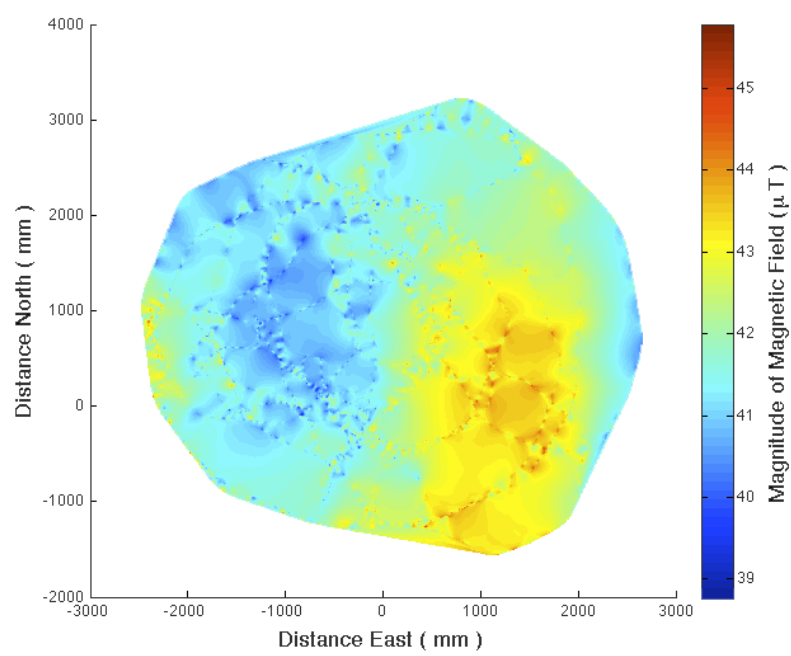

Fig. 8. Heatmap of Magnetic Field of the Motion Lab.

Reference measurements have been made by a Qualisys system. This technology provides quaternions with a precision of $0.5^{\circ}$ of rotation. Our room is equipped with 20 Oqus cameras connected to a server and Qualisys Tracker software. A $150 \mathrm{~Hz}$ sampling is used. For the purpose of aligning timestamps of our ground truth data with smartphone's sensors data, we used slerp interpolation [21]. The motion tracker reference frame has been aligned with EF thanks to DGPS measures provided by room architects. A smartphone handler with infrared markers has been created with a 3D printer for this study. Smartphone handler markers have been aligned with SF (Fig. 9).

Most of smartphones motion APIs (Android, iOS, Windows Phone) provide calibrated data. It is also possible to get

3 https://github.com/sensbio/sensors-monitoring-android

${ }^{4}$ See: http://kinovis.inrialpes.fr 


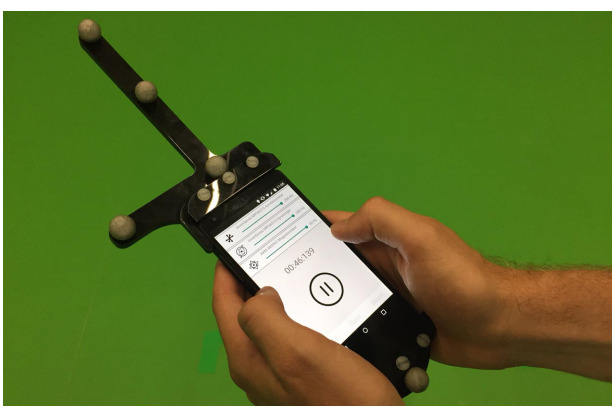

Fig. 9. Smartphone handler for Motion Lab with homemade recording app.

uncalibrated data and apply custom algorithms, as we do in this paper. Following results come from accelerometer calibration from Frosio [17] and magnetometer calibration from Renaudin [19].

\section{B. Results, analyzes and discussions}

In order to have a variety of different handheld motions datasets, we identify five typical motions for a smartphone, inspired from [22]:

Walking with a texting hand: the user is walking while typing or reading a message/instruction on the screens device.

Walking with a phoning hand: the user is using the device to make or receive a phone call.

Walking with a swinging hand: the user is walking holding the portable device in the hand.

Walking with the mobile device in the back pocket.

Static: the subject is not moving.

We reported on precision error using the mean absolute error (MAE) and the standard deviation (STD) on yaw, pitch and roll angles. In order to avoid errors due to gimbal-lock, we also used the quaternion angle difference (QAD) [23] defined by:

$$
\theta=\cos ^{-1}\left(2\langle q 1, q 2\rangle^{2}-1\right)
$$

The Android API of our Nexus 5 also provides quaternions. We added them to our results even if we do not know about the "black-box algorithm" that generated them. We assume that they have an "on-the-fly" calibration. We also assume that this calibration was used for data provided by the API. Results are split in five parts. The first part is a review of results obtained with compared algorithms. The second one is a comparison between motions. Then the third part shows the importance of calibration. A part summarizes a comparison of algorithms in the presence of magnetic deviations. Finally the last part reports on processing time spent in each algorithm.

This paper reports only on a fraction of the results that we have obtained. More extensive results are available from http://tyrex.inria.fr/mobile/benchmarks-attitude

1) Comparison of results with different algorithms: Table III reports the MAE and STD for the first four motions (texting, phoning, swinging, pocket) for a duration of $180 \mathrm{~s}$. An example of absolute error using QAD during the phoning motion is shown in Fig. 10. Fourati and Martin's algorithms are the two best in terms of overall comparison. Fourati's one is quiet good during low accelerations, but it is slightly better than others algorithms during high accelerations, more details will be brings in next section. Renaudin's algorithm is resulting from a recent work and its behavior is the best during low accelerations. More adjustments about QSF detectors may make it one of the best. We can also conclude that sensors' biases and noises consideration described in Table I have not an important impact on results.

TABLE III. COMPARISON OF ATTITUDE ESTIMATION VIA EACH ALGORITHM.

\begin{tabular}{l|l|l|l|l|l|l|l|l|} 
& \multicolumn{2}{|c|}{ QAD } & \multicolumn{2}{c|}{ Yaw } & \multicolumn{2}{c|}{ Pitch } & \multicolumn{2}{c|}{ Roll } \\
& MAE & STD & MAE & STD & MAE & STD & MAE & STD \\
\hline Android & $9.47^{\circ}$ & $5.15^{\circ}$ & $11.4^{\circ}$ & $15.2^{\circ}$ & $1.32^{\circ}$ & $0.98^{\circ}$ & $4.90^{\circ}$ & $7.07^{\circ}$ \\
\hline Mahony & $5.26^{\circ}$ & $4.57^{\circ}$ & $8.54^{\circ}$ & $12.9^{\circ}$ & $2.16^{\circ}$ & $2.34^{\circ}$ & $7.64^{\circ}$ & $10.7^{\circ}$ \\
\hline Madgwick & $6.05^{\circ}$ & $5.53^{\circ}$ & $7.58^{\circ}$ & $12.3^{\circ}$ & $2.33^{\circ}$ & $2.38^{\circ}$ & $9.03^{\circ}$ & $9.72^{\circ}$ \\
\hline Fourati & $4.72^{\circ}$ & $2.44^{\circ}$ & $6.92^{\circ}$ & $9.97^{\circ}$ & $1.24^{\circ}$ & $1.12^{\circ}$ & $5.66^{\circ}$ & $8.19^{\circ}$ \\
\hline Choukroun & $5.50^{\circ}$ & $2.44^{\circ}$ & $9.06^{\circ}$ & $12.4^{\circ}$ & $2.84^{\circ}$ & $3.21^{\circ}$ & $9.19^{\circ}$ & $12.4^{\circ}$ \\
\hline Martin & $4.80^{\circ}$ & $2.52^{\circ}$ & $8.37^{\circ}$ & $11.8^{\circ}$ & $2.11^{\circ}$ & $2.62^{\circ}$ & $8.83^{\circ}$ & $12.1^{\circ}$ \\
\hline Renaudin & $5.40^{\circ}$ & $2.14^{\circ}$ & $6.20^{\circ}$ & $6.96^{\circ}$ & $1.47^{\circ}$ & $1.62^{\circ}$ & $3.61^{\circ}$ & $4.48^{\circ}$ \\
\hline
\end{tabular}

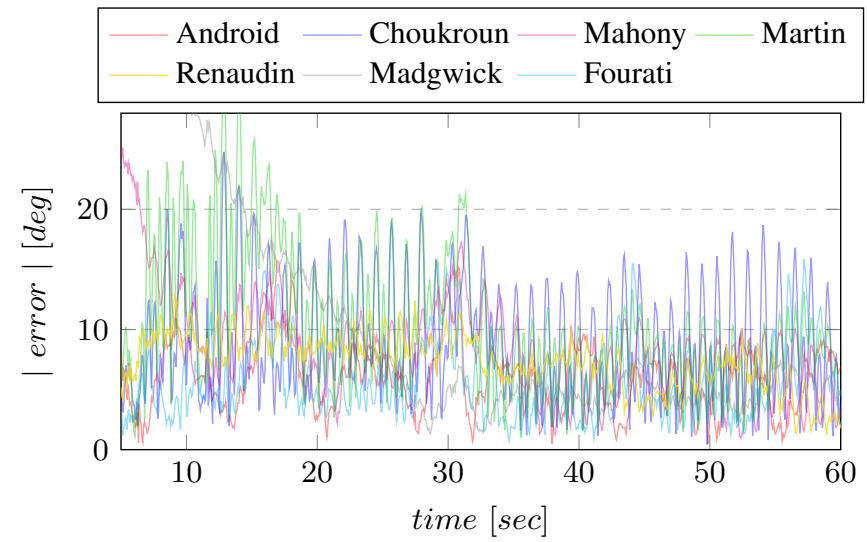

Fig. 10. Precision error on quaternion components during a swinging motion.

2) Motions: Based on our datasets, we also provide a comparison about the precision of attitude estimation with respect to each motion (Table. IV). We also correlate these results with the variance of the acceleration norm because most of the algorithms do not consider external acceleration (as discussed in Section [III). STD is reported in Table V

The more acceleration deviates from $g$, the worst the attitude estimation is. For example swinging motion and back pocket (due to hip movements) are harder to estimate. However, Fourati's solution improves the estimation by adjusting the filter gain according to large external accelerations. Moreover, results with the back pocket motion confirm the importance of QAD to calculate the precision of estimation when the smartphone is put in a vertical position prone to the gimbal-lock problem. Therefore we cannot directly use the yaw extracted from the quaternion to determine the heading in a PDR solution when the smartphone is put vertically.

3) Calibration: Previous results with the four typical motions used accelerometer calibration from Frosio [17] and magnetometer calibration from Renaudin [19]. The Android API also provides its own calibration for these two sensors. Table VI reports on the mean MAE on QAD using uncalibrated data, Android calibrated data and Frosio/Renaudin calibrated data, for all algorithms. 
TABLE IV. COMPARISON OF ATTITUDE ESTIMATION ACCORDING TO TYPICAL MOTIONS.

\begin{tabular}{l|l|l|l|l|l|l|l|l|} 
& \multicolumn{2}{|c|}{ QAD } & \multicolumn{2}{c|}{ Yaw } & \multicolumn{2}{c|}{ Pitch } & \multicolumn{2}{c|}{ Roll } \\
& MAE & STD & MAE & STD & MAE & STD & MAE & STD \\
\hline Texting & $3.88^{\circ}$ & $3.16^{\circ}$ & $2.91^{\circ}$ & $4.18^{\circ}$ & $0.99^{\circ}$ & $1.10^{\circ}$ & $1.40^{\circ}$ & $1.69^{\circ}$ \\
\hline Phoning & $4.24^{\circ}$ & $2.59^{\circ}$ & $2.84^{\circ}$ & $3.32^{\circ}$ & $1.42^{\circ}$ & $1.56^{\circ}$ & $2.45^{\circ}$ & $2.43^{\circ}$ \\
\hline Pocket & $6.05^{\circ}$ & $3.38^{\circ}$ & $21.5^{\circ}$ & $32.2^{\circ}$ & $1.65^{\circ}$ & $1.81^{\circ}$ & $22.4^{\circ}$ & $31.4^{\circ}$ \\
\hline Swinging & $7.50^{\circ}$ & $3.96^{\circ}$ & $3.85^{\circ}$ & $4.62^{\circ}$ & $4.06^{\circ}$ & $4.40^{\circ}$ & $3.09^{\circ}$ & $3.60^{\circ}$ \\
\hline
\end{tabular}

TABLE V. STD OF ACCELERATION NORM IN THE FOUR MOTIONS IN m.s $s^{-2}$

\begin{tabular}{c|c|c|c} 
Texting & Phoning & Swinging & Back pocket \\
\hline 0.902 & 0.779 & 1.890 & 1.741 \\
\hline
\end{tabular}

Uncalibrated data are unusable for attitude estimation algorithms. This is due to the fact that the internal speaker is close to IMU $(\sim 2 \mathrm{~cm})$ inside the smartphone. In a context free from magnetic deviations, the mean of uncalibrated magnetic field magnitude is about $350 \mu T$. This bias can easily be removed with a calibration of hard iron deviations (same frame). The magnetometer calibration given by Android API is enough to have a good attitude estimation in most cases. Since this calibration is a "black-box" and the system calibrates on the fly, sometimes there is unwanted behavior (like in our phoning dataset where $M A E=20^{\circ}$ ), while Renaudin's calibration gives a MAE less than $4^{\circ}$. In our datasets, Renaudin's calibration improves the quality of attitude estimation by $4.5^{\circ}$ compared to Android's one. This improvement is significant. Finally, adding the Frosio calibration improves the global precision by $0.8^{\circ}$, event if this calibration is often tedious to realize.

TABLE VI. COMPARISON OF ATTITUDE ESTIMATION ACCORDING TO CALIBRATION.

\begin{tabular}{c|c|c|c|c|} 
& & & & Renaud. and \\
& Uncalibrated & Android calib. & Renaud. calib. & Frosio calib. \\
\hline MAE & $92.64^{\circ}$ & $10.53^{\circ}$ & $6.05^{\circ}$ & $5.27^{\circ}$ \\
\hline STD & $41.13^{\circ}$ & $6.41^{\circ}$ & $3.58^{\circ}$ & $3.50^{\circ}$ \\
\hline
\end{tabular}

4) Magnetic deviation: During the static phase we chose to test the algorithm resiliency to magnetic deviations on yaw, pitch and roll components. We used 6 small magnets and put them close to the smartphone at $19 \mathrm{sec}$ and then removed them at $25 \mathrm{sec}$. The magnetometer reported a magnitude of $250 \mu \mathrm{T}$ during the period instead of $45 \mu T$ for a free space (see Fig. 11.

In the static phase, after a certain convergence period, all algorithms provides a stable but different estimation of attitude. In order to discard these differences from our error measurements, we first substract the mean attitude estimation for each dataset. Figs. 12, 13, 14 report the behaviors of the six algorithms during the magnetic deviation on QAD, yaw, pitch and roll. Scores on MAE and STD are shown in Table VII

The only algorithms that are not impacted (or very little) by the magnetic deviation during this static phase are Renaudin's and Android ones. For Renaudin's algorithm, this is thanks to the detection of a high variation in the norm of the magnetic field, which is then used to discard magnetometer values in their Kalman Filter [1]. Martin's technique with the cross product of acceleration and magnetic field allows to avoid the distortions on pitch and roll. Convergence of Fourati's filter is much faster than with other algorithms (convergence delay is less than $1 s$ after the magnet is removed). Moreover the pitch and roll angles are not as significantly impacted as with the other approaches.

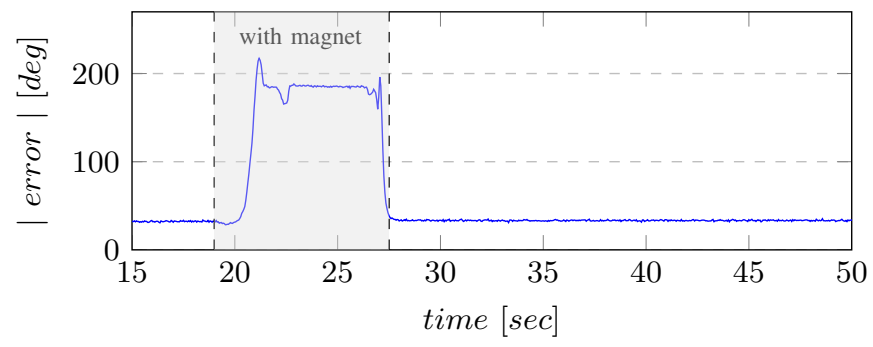

Fig. 11. Magnitude of magnetic field using a magnet.

TABLE VII. COMPARISON OF ATTITUDE ESTIMATION UNDER MAGNETIC DEVIATION PROVIDED BY EACH ALGORITHM.

\begin{tabular}{l|l|l|l|l|l|l|l|l|} 
& \multicolumn{2}{|c|}{ QAD } & \multicolumn{2}{c|}{ Yaw } & \multicolumn{2}{c|}{ Pitch } & \multicolumn{2}{c|}{ Roll } \\
& MAE & STD & MAE & STD & MAE & STD & MAE & STD \\
\hline Android & $0.01^{\circ}$ & $0.01^{\circ}$ & $0.03^{\circ}$ & $0.01^{\circ}$ & $0.02^{\circ}$ & $0.01^{\circ}$ & $0.06^{\circ}$ & $0.03^{\circ}$ \\
\hline Mahony & $17.1^{\circ}$ & $30.3^{\circ}$ & $17.2^{\circ}$ & $30.3^{\circ}$ & $0.39^{\circ}$ & $0.61^{\circ}$ & $0.56^{\circ}$ & $1.18^{\circ}$ \\
\hline Madgwick & $22.0^{\circ}$ & $24.8^{\circ}$ & $22.0^{\circ}$ & $24.8^{\circ}$ & $0.44^{\circ}$ & $0.65^{\circ}$ & $1.37^{\circ}$ & $2.06^{\circ}$ \\
\hline Fourati & $14.6^{\circ}$ & $27.6^{\circ}$ & $14.7^{\circ}$ & $27.6^{\circ}$ & $0.03^{\circ}$ & $0.05^{\circ}$ & $0.15^{\circ}$ & $0.23^{\circ}$ \\
\hline Choukroun & $14.2^{\circ}$ & $14.9^{\circ}$ & $14.3^{\circ}$ & $15.0^{\circ}$ & $0.36^{\circ}$ & $0.63^{\circ}$ & $1.29^{\circ}$ & $2.23^{\circ}$ \\
\hline Martin & $20.2^{\circ}$ & $26.2^{\circ}$ & $20.1^{\circ}$ & $26.2^{\circ}$ & $0.01^{\circ}$ & $0.01^{\circ}$ & $0.02^{\circ}$ & $0.03^{\circ}$ \\
\hline Renaudin & $0.55^{\circ}$ & $0.25^{\circ}$ & $0.48^{\circ}$ & $0.25^{\circ}$ & $0.01^{\circ}$ & $0.01^{\circ}$ & $0.03^{\circ}$ & $0.03^{\circ}$ \\
\hline
\end{tabular}

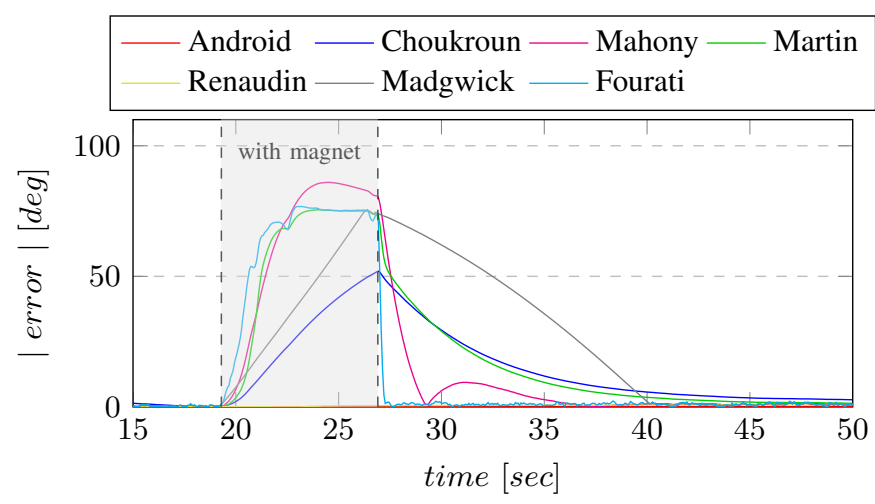

Fig. 12. Precision error on yaw component with a magnet distortion.

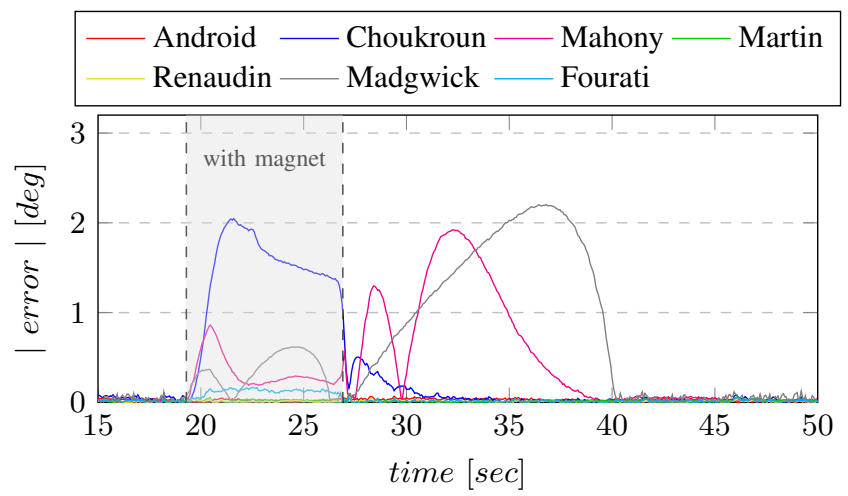

Fig. 13. Precision error on pitch component with a magnet distortion. 


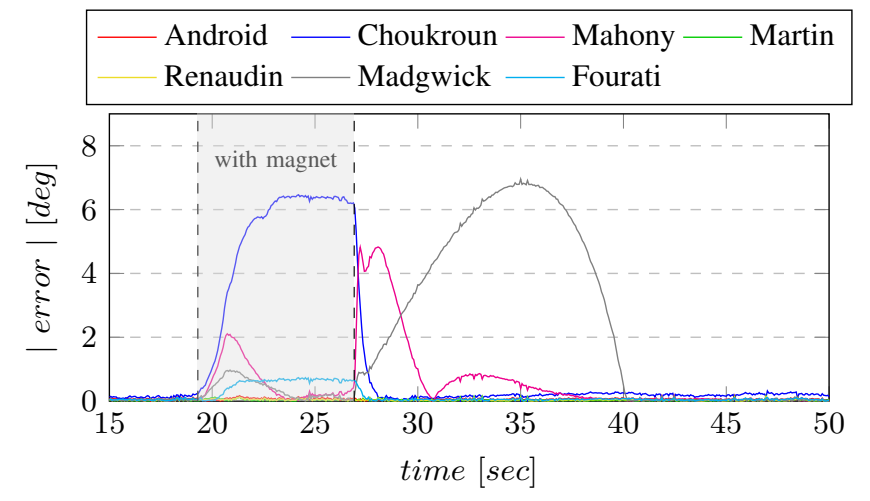

Fig. 14. Precision error on roll component with a magnet distortion.

5) Processing time: Since the device is a smartphone and battery saving is crucial, processing time matters. Table VIII reports the number of quaternions generated per second using Matlab. Computationally, the Madgwick filter outperforms the others. The second line of the Table thus indicates the ratio of the time spent in each algorithm relative to the fastest (Madgwick).

TABLE VIII. PRocessing TIME ON QUATERNION GENERATION (QUAT/SEC)

\begin{tabular}{c|c|c|c|c|c|} 
& Mahony & Madgwick & Fourati & Choukroun & Martin \\
\hline Quaternion gen./sec & 2762 & 4052 & 2559 & 2148 & 1257 \\
\hline Relative to the best & 0.68 & 1 & 0.63 & 0.53 & 0.31 \\
\hline
\end{tabular}

Overall, in our setting, the two most suited algorithms are the Martin's observer and the complementary filter from Fourati. The first one is a bit more robust to magnetic deviations thanks to the cross product of acceleration and magnetic field but its precision is limited for high accelerations. In this situation, the simple dynamic gain from Fourati's algorithm shows good results. Other solutions like Madgwick one's might also be especially interesting when the battery life is a priority. Some of the best ideas like Renaudin's QSF or dynamic gains from Fourati will be the subject of our future researches towards improved algorithms.

\section{COnClusions}

One major difficulty in PDR algorithms resides in precise attitude estimation in the presence of magnetic deviations. Several techniques have been developed in the literature for different scenarios. We provided an experimental setup with a precise ground truth obtained through a motion capture system. We precisely quantified the attitude estimation error obtained with each technique. We analyzed and discussed the results that we have obtained.

\section{ACKNOWLEDGMENTS}

This work has been partially supported by the LabEx PERSYVAL-Lab (ANR11-LABX-0025), EquipEx KINOVIS (ANR-11-EQPX-0024), EquipEx Robotex (ANR10-EQPX-4401). We thank J.F. Cuniberto for the smartphone handler, J. Dumon and M. Heudre for the help in designing the experimental platform and C. Combettes to provide Renaudin's results.

\section{REFERENCES}

[1] V. Renaudin and C. Combettes, "Magnetic, acceleration fields and gyroscope quaternion (MAGYQ)-based attitude estimation with smartphone sensors for indoor pedestrian navigation," Sensors, vol. 14, no. 12, pp. 22 864-22 890, 2014.

[2] L. E. Miller, Indoor navigation for first responders: a feasibility study. Citeseer, 2006.

[3] M. Razafimahazo, N. Layaïda, P. Genevès, and T. Michel, "Mobile augmented reality applications for smart cities," ERCIM News 98, no. 98, pp. 45-46, Jul. 2014.

[4] R. Mautz, "Indoor positioning technologies," Ph.D. dissertation, Habilitationsschrift ETH Zürich, 2012.

[5] J. L. Crassidis, F. L. Markley, and Y. Cheng, "Survey of nonlinear attitude estimation methods," Journal of Guidance, Control, and Dynamics, vol. 30, no. 1, pp. 12-28, 2007.

[6] D. Choukroun, I. Y. Bar-Itzhack, and Y. Oshman, "Novel quaternion kalman filter," Aerospace and Electronic Systems, IEEE Transactions on, vol. 42, no. 1, pp. 174-190, 2006.

[7] R. Mahony, T. Hamel, and J.-M. Pflimlin, "Nonlinear complementary filters on the special orthogonal group," Automatic Control, IEEE Transactions on, vol. 53, no. 5, pp. 1203-1218, 2008.

[8] S. O. Madgwick, A. J. Harrison, and R. Vaidyanathan, "Estimation of IMU and MARG orientation using a gradient descent algorithm," in Rehabilitation Robotics (ICORR), 2011 IEEE International Conference on. IEEE, 2011, pp. 1-7.

[9] H. Fourati, "Heterogeneous data fusion algorithm for pedestrian navigation via foot-mounted inertial measurement unit and complementary filter," Instrumentation and Measurement, IEEE Transactions on, vol. 64, no. 1, pp. 221-229, Jan 2015.

[10] P. Martin and E. Salaün, "Design and implementation of a lowcost observer-based attitude and heading reference system," Control Engineering Practice, vol. 18, no. 7, pp. 712-722, 2010.

[11] M. H. Afzal, V. Renaudin, and G. Lachapelle, "Magnetic field based heading estimation for pedestrian navigation environments," in Indoor Positioning and Indoor Navigation (IPIN), 2011 International Conference on. IEEE, 2011, pp. 1-10.

[12] D. Sachs, "Sensor fusion on android devices: A revolution in motion processing," [Video] https://www.youtube.com/watch?v= C7JQ7Rpwn2k. 2010, [Online; accessed 9-April-2015].

[13] J. B. Kuipers, Quaternions and rotation sequences, 1999, vol. 66.

[14] J. Diebel, "Representing attitude: Euler angles, unit quaternions, and rotation vectors," Matrix, vol. 58, pp. 15-16, 2006.

[15] X. Zhang, Y. Li, P. Mumford, and C. Rizos, "Allan variance analysis on error characters of MEMS inertial sensors for an FPGA-based GPS/INS system," in Proceedings of the International Symposium on GPS/GNNS, 2008, pp. 127-133.

[16] H. Hou, Modeling inertial sensors errors using Allan variance. University of Calgary, Department of Geomatics Engineering, 2004.

[17] I. Frosio, F. Pedersini, and N. A. Borghese, "Autocalibration of MEMS accelerometers," in Advanced Mechatronics and MEMS Devices. Springer, 2013, pp. 53-88.

[18] U. (NGA) and the U.K.'s Defence Geographic Centre (DGC), "The world magnetic model," http://www.ngdc.noaa.gov/geomag/WMM 2015, [Online; accessed 17-July-2015].

[19] V. Renaudin, M. H. Afzal, and G. Lachapelle, "New method for magnetometers based orientation estimation," in Position Location and Navigation Symposium (PLANS), 2010 IEEE/ION. IEEE, 2010, pp. 348-356.

[20] P. Bartz, "Magnetic compass calibration," Oct. 13 1987, US Patent $4,698,912$.

[21] K. Shoemake, "Animating rotation with quaternion curves," in ACM SIGGRAPH computer graphics, vol. 19, no. 3. ACM, 1985, pp. 245254.

[22] V. Renaudin, M. Susi, and G. Lachapelle, "Step length estimation using handheld inertial sensors," Sensors, vol. 12, no. 7, pp. 8507-8525, 2012.

[23] D. Q. Huynh, "Metrics for 3D rotations: Comparison and analysis," Journal of Mathematical Imaging and Vision, vol. 35, no. 2, pp. 155164, 2009. 